\title{
Evaluation of Efficacy and Safety of Premixed Parenteral Nutrition Versus Customized Parenteral Nutrition in a Large Teaching Hospital
}

\author{
Teresa Pounds $^{1}$, Annesha Lovett ${ }^{2, *}$, Susiana Eng $^{2}$, Khalid Iqbal $^{3}$, Israel Orija ${ }^{4}$, Jessica Chmielewski ${ }^{5}$ \\ ${ }^{1}$ BCNSP Atlanta Medical Center, Department of Pharmacy 303 Parkway Drive Northeast, Atlanta, GA 30312 \\ ${ }^{2}$ Mercer University, College of Pharmacy \& Health Sciences ,3001 Mercer University Drive, Atlanta, GA 30341-4155 \\ ${ }^{3}$ Atlanta South Nephrology, 1275 Cleveland Avenue, East Point, GA 30344 \\ ${ }^{4}$ Atlanta Medical Center, Department of Endocrinology, 303 Parkway Drive Northeast, Atlanta, GA 30312 \\ ${ }^{5}$ Cartersville Medical Center ,Department of Pharmacy,960 Joe Frank Harris Pkwy SE , Cartersville, GA 30120 \\ *Corresponding Author: lovett_aw@mercer.edu
}

Copyright $(2013$ Horizon Research Publishing All rights reserved.

\begin{abstract}
In the last two decades, there has been a significant degree of controversy surrounding the use of standardized parenteral solutions. Opponents of standardized parenteral nutrition claim that individualized parenteral nutrition is necessary because the critically ill patient requires daily manipulation of macro- and micro-nutrients secondary to stress associated with complex ailments. The purpose of this study was to determine if premixed parenteral nutrition solutions were equally safe and efficacious, while being less costly, in managing parenteral nutrition of patients at a large metropolitan teaching hospital, as compared to customized parenteral nutrition solutions. This was a randomized controlled trial with no placebo involving 100 patients requiring central parenteral nutrition. Consenting patients received either customized parenteral nutrition or premixed double chamber parenteral nutrition containing protein, carbohydrates and electrolytes. Vitamins and trace elements were added as needed. Each subject enrolled in the study was followed until the parenteral nutrition was either discontinued, the patient was transferred from the facility, or the patient expired during the study. Premixed and customized parenteral nutrition solutions were shown to be equally efficacious in patients as their blood glucose, fluid status, nutritional markers, serum electrolytes, phosphate, and magnesium values were within normal limits $(\mathrm{p}<.05)$. Premixed parenteral nutrition is associated with significantly better safety as compared to customized parenteral nutrition [11]. Furthermore, the cost difference was $\$ 41,934$ when comparing the premixed formulation to the customized solution. In this study, premixed parenteral nutrition solutions were equally safe, effective, and less costly than customized parenteral nutrition formulas. Patients receiving premixed parenteral nutrition experienced less adverse drug reactions and metabolic abnormalities than patients receiving customized parenteral nutrition.
\end{abstract}

Keywords Parenteral Formulas/Compounding, Nutrition, Parenteral Nutrition

\section{Background}

Parenteral nutrition $(\mathrm{PN})$ solutions are utilized to ensure an adequate delivery of calories and protein when patients are unable to take sufficient food by either the oral route or via feeding tube. PN is utilized in a variety of patient populations, including surgical patients, severely malnourished patients, and patients in the intensive care unit. USP <797> standards have to be followed when compounding solutions. The premixed solutions require fewer preparations than compounded products minimizing the likelihood of mistakes and contamination. In 2003, the Joint Commission developed stipulations that drug concentrations be standardized and limited in number as part of the Joint Commission National Patient Safety Goals of 2003.[1] In response to this mandate, the American Society for Parenteral and Enteral Nutrition (A.S.P.E.N.) reviewed the published literature for the use of a standardized PN formulation to recommend to hospitals as a commercially prepared, premixed formulation.[2] A.S.P.E.N. also recognized the need that hospitals continue to have the ability to use customized, compounded PN formulations, after ensuring that the compounding process becomes standardized and adheres to national recommendations. However, no recommendation was made on whether hospitals should preferentially use commercially, premixed formulations or compounded PN formulations.[2]

In the last two decades, there has been a discussion regarding the advantages and utilization of standardized 
parenteral solutions in the United States. Commercial, premixed nutritional solutions are formulated with standard amounts of calories and electrolytes that meet the general nutrition requirements of a patient. Recent studies have found that the commercial, premixed formulations are at least as effective as compounded formulations in controlling serum electrolytes in both adult and pediatric populations. [3, $4,5]$ One study examined the safety and cost of receiving commercially prepared premixed formulations compared to compounded PN solutions. [6, 7] The study determined that there was a $30 \%$ greater probability of developing a bloodstream infection with compounded parenteral nutrition solution compared to a premixed formulation $(\mathrm{p}<0.0001)$. [6] Upon further analysis, it was determined that there were fewer associated adverse events (i.e. bacterial infection, pneumonia, blood infection, intra-abdominal infection, skin/soft tissue infection, urinary tract infection, sepsis, severe sepsis, and septic shock) with the commercial, premixed formulation compared to the compounded PN solution (observed $17.5 \%$ vs. $26.6 \%, p<0.001$ ). [7] It was also determined that the average acquisition cost for a premixed formulation was $\$ 164$ compared to $\$ 239$ for a compounded PN solution. [6]

Other advantages of commercial, premixed formulations include ease of prescribing and preparation, potential lower costs, and reduced risk for microbial contamination. $[8,9]$ Disadvantages of commercial, premixed formulations include the limitations in availability of products in the United States (currently only a 2-compartment premixed product is available), use in critically ill or obese patients who require higher daily protein requirements, inability to allow for changes to electrolytes, and additional education sessions required for prescribers on how to prescribe and order premixed products. $[8,9,10]$

The primary objective of this study is to examine whether commercially prepared premixed formulations are as safe and efficacious as compounded, customized PN solutions at a large, metropolitan, teaching hospital. The secondary objective of this study is to examine the costs associated with customized PN solutions and commercially prepared premixed formulations.

\section{Methods}

This randomized controlled trial was completed at Atlanta Medical Center, a 460-bed tertiary care hospital in Atlanta Georgia. The study was conducted from August 2008 to August 2011 and comprised of 100 patients who required central parenteral nutrition. After obtaining informed consent, patients were placed either in the control group (customized PN) or in the treatment group (premixed PN) using a randomization chart. A total of 50 patients were randomized to each group.

Due to the nature of the study, blinding was not possible since the prescribing clinician needed to be aware of the formulations prior to ordering. Inclusion criteria were all patients requiring parenteral nutrition support according to the A.S.P.E.N. approved indications for parenteral nutrition use. Additionally, patients had to be 18-years of age or older, receiving central PN for duration of at least 3 days as the sole source of nutrition. All patients were either from the Intensive Care Unit (ICU) or Medical/Surgical patient care units.

Exclusion criteria were patients receiving home PN, patients receiving a combination of $\mathrm{PN}$ as well as enteral feedings, patients who switched from customized PN to premixed PN formulations and vice versa, patients receiving peripheral PN, patients with a serum creatinine of 1.5 or greater, and patients requiring fluid restriction of less than 2 liters per day. Each study subject enrolled in the study was followed until the PN was either discontinued, the patient was transferred from the facility, or the patient expired.

Table 1. Premixed Clinimix formulations that were used in the study

\begin{tabular}{|c|c|c|}
\hline Product & Clinimix E 5/15 & Clinimix E 5/20 \\
\hline Total Volume & 2 liters & $5 \%$ \\
\hline Amino Acid Concentration & $5 \%$ & $20 \%$ \\
\hline Dextrose Concentration & $15 \%$ & 400 \\
\hline Gram Dextrose/ 2L & 300 & 100 \\
\hline Gram Amino Acids/ 2L & 100 & 70.52 \\
\hline Gram Nitrogen/ 2L & 16.52 & 60 \\
\hline Sodium (mEq/ 2L) & 70 & 10 \\
\hline Potassium (mEq/ 2L) & 60 & 9 \\
\hline Magnesium (mEq/ 2L) & 10 & 160 \\
\hline Calcium (mEq/ 2L) & 9 & 78 \\
\hline Acetate (mEq/ 2L) & 160 & 30 \\
\hline Chloride (mEq/ 2L) & 78 & 6.0 \\
\hline Phosphate (mmol/ 2L) & 30 & 1650 \\
\hline $\mathrm{pH}$ & 6.0 & 400 \\
\hline Osmolarity & 1395 & 1360 \\
\hline Kcal from Amino Acids/ 2L & 400 & 1760 \\
\hline kCal from Dextrose/ 2L & 1020 & \\
\hline Total Kcal/ 2L & 1420 & \\
\hline
\end{tabular}


Patients received either customized PN or premixed double chamber PN containing protein, carbohydrate, and electrolytes (Table 1). The study product used was Clinimix Premixed PN Solution. A standardized electrolyte profile included in the Clinimix Premixed PN bags falls within the ranges that ASPEN recommends for non-acute patients in the United States. Atlanta Medical Center estimated that about $70 \%$ of its adult PN orders would be Clinimix E formulas (i.e. premixed PN with electrolytes present) while the remaining $30 \%$ of adult parenteral nutrition orders would be Clinimix Plain (i.e. parenteral nutrition without electrolytes present) with the utilization of fat emulsions via piggyback route.

The PN formulations of the two groups were equivalent as it pertains to caloric, protein, carbohydrate, and electrolyte content. The method for compounding formulations was that components were added manually. Vitamins and trace elements were added as needed to all PN orders. Patients who needed fat emulsions infused had fat emulsions co-infused via a y-site. The reference product used in the control group was customized parenteral nutrition solution with piggyback fat emulsions. Each subject enrolled in the study was followed, and the patient's metabolic panel, magnesium, phosphate, and calcium levels were monitored and noted until the parenteral nutrition was discontinued, patient was transferred from the facility, or the patient expired.

Metabolic markers (e.g. C-reactive protein) were assessed at baseline and every seven days per Atlanta Medical Center central parenteral nutrition protocol. All patients had serum electrolytes collected at baseline and daily (i.e. $\mathrm{K}, \mathrm{Na}, \mathrm{CO} 2$, $\mathrm{Cl}, \mathrm{BUN}, \mathrm{SrCr}$ ). Electrolytes were administered as boluses if the serum concentrations were low. Serum glucose was taken every 6 hours until the patient was metabolically stabilized and then glucose was taken only once a day. Serum chemistries to assess liver function were also monitored at baseline and then every Monday and Thursday (i.e. Bilirubin, AST, ALT and Alkaline phosphatase). Hematocrit and white blood counts were utilized for hematological monitoring.

Any changes that were made to a patient's parenteral nutrition formulas including electrolyte adjustments, changes in a patient's medical condition, nutritional status, fluid needs, glycemic changes, or transition from parenteral or enteral were well documented on a daily basis. Documentation was also made when there were changes to electrolytes, changes in patient's medical conditions, changes in nutritional status, changes in fluid needs, transitioning from parenteral nutrition to oral diet, transitioning from parenteral nutrition to enteral tube feeding, and glycemic changes. Infectious complications such as sepsis or microbiologically confirmed infections were recorded for both groups in the study. Infection sites were classified as bloodstream infections, pneumonia, skin and soft tissue infections, urinary tract infections, and intra-abdominal infections.

Errors and adverse events were reported using the
"Adverse Drug Reaction (ADR) Form" obtained from the Atlanta Medical Center ADR reporting policy. Safety goals were measured through the examination of the ADR reports. It should be noted that the incidence of adverse drug reaction was not based on the severity of illness or the period of time that a patient was receiving parenteral nutrition. Efficacy was measured by serum electrolytes, calcium, phosphate, and magnesium values being within normal limits and the increasing trend in prealbumin. Costs were determined by calculating the difference between the charges to Atlanta Medical Center for ingredients in the premixed double chamber bags versus the charges for the ingredients in the customized parenteral nutrition. Cost data was not adjusted for increases in health care cost of living and reflects data collected specifically for this study. Descriptive statistics was used to analyze the data and the chi square test was used to compare proportions. Data are presented as means \pm SD. Statistical significance was set at $\mathrm{p}<0.05$. The study was approved by the Atlanta Medical Center Institutional Review Board.

\section{Results}

There were a total of 100 patients that participated in the study. Of the 140 patients who were eligible to be in the study 40 did not consent to participate. The average age was $50 \pm 13.95$ years for the premixed PN (treatment group) and $53 \pm 18.59$ years for the customized PN (control group). Body mass index (BMI) was similar between groups with values of $25.28 \mathrm{~m}^{2} \pm 10.20$ and $24.83 \mathrm{~m}^{2} \pm 5.78$ for the treatment and control groups, respectively, while total length of stay was $23 \pm 16.80$ and $21 \pm 13.19$ days for treatment and control groups, respectively (Table 2). There were no statistically significant differences between groups with respect to patient baseline characteristics.

The chi-square test of independence was used to examine the efficacy of premixed PN by comparing the proportion of normal lab tests between treatment and control groups. Analysis by chi-square test was significant $(\mathrm{p}<.05)$ with patients receiving premixed PN having serum electrolytes, phosphate, and magnesium values that were within normal limits. Commercially prepared premixed formulations were shown to be as efficacious as compounded, customized PN solutions (Table 3).

The ADR report revealed that $44 \%$ of the patients receiving premixed PN did not develop an adverse drug reaction as compared to $24 \%$ of patients receiving customized PN. The top five types of infection that premixed and customized PN patients developed were intra-abdominal infection ( $30 \%$ vs. $40 \%$ ), bacterial infection ( $16 \%$ vs. $32 \%$ ), severe sepsis (12\% vs. $18 \%$ ), urinary tract infection (10\% vs. $16 \%)$ and skin/soft tissue infection (6\% vs. $10 \%)$, respectively (Figure 1).

Finally, costs associated with customized PN solutions and commercial premixed formulations were explored by calculating the difference between the charges for 
ingredients. Actual cost data was not available therefore hospital charge data was utilized. The one year charge was $\$ 242,178$ for the ingredients in the premixed formulation and $\$ 284,112$ for the ingredients in the customized PN solution resulting in a difference of $\$ 41,934$ (Figure 2). It should be noted that charges were extrapolated for a one year period and the charge represents the charge to the hospital for supplies to the patients in the study.

Table 2. Patient Demographics

\begin{tabular}{cccc}
\hline & Treatment Group & \multicolumn{2}{c}{ Control Group } \\
Customized (C) \\
& Premixed (P) & \\
$\mathrm{n}=50$ & $50.84 \pm 13.95$ & $53.78 \pm 18.59$ & .37 \\
P Value
\end{tabular}

$\mathrm{P}=$ Premixed

$\mathrm{C}=$ Customized

BMI (Body mass index) is defined as the individual's body mass divided by the square of their height ( $\mathrm{m}=\mathrm{meter}$ ). $\mathrm{p}<.05$, statistically significant

Table 3. Premixed vs. customized laboratory test results

\begin{tabular}{cccccccccccccccccc}
\hline $\mathrm{Na}$ & & $\mathrm{K}$ & & $\mathrm{CO}$ & & $\mathrm{Glu}$ & & $\mathrm{Ca}$ & & $\mathrm{P}$ & & $\mathrm{M}$ & & \multicolumn{2}{c}{ Total } \\
\hline $\mathrm{P}$ & $\mathrm{C}$ & $\mathrm{P}$ & $\mathrm{C}$ & $\mathrm{P}$ & $\mathrm{C}$ & $\mathrm{P}$ & $\mathrm{C}$ & $\mathrm{P}$ & $\mathrm{C}$ & $\mathrm{P}$ & $\mathrm{C}$ & $\mathrm{P}$ & $\mathrm{C}$ & $\mathrm{P}$ & $\mathrm{C}$ \\
\hline 528 & 515 & 528 & 515 & 528 & 515 & 528 & 515 & 528 & 515 & 238 & 230 & 262 & 238 & 3140 & 3043 \\
\hline 397 & 360 & 473 & 444 & 473 & 410 & 249 & 182 & 147 & 152 & 186 & 158 & 238 & 228 & 2163 & 1934 \\
\hline 131 & 155 & 55 & 71 & 55 & 105 & 279 & 333 & 381 & 363 & 52 & 72 & 24 & 10 & 977 & 1109 \\
25 & 52 & $1 \mathrm{C}$ & 22 & 38 & 41 & 251 & 319 & 4 & 9 & 15 & 21 & 4 & 5 & 347 & 469 \\
106 & 103 & 45 & 49 & 17 & 64 & 28 & 14 & 377 & 354 & 37 & 51 & 20 & 5 & 630 & 640 \\
\hline
\end{tabular}

\section{$\mathrm{P}=$ Premixed}

$\mathrm{C}=$ Customized

Calculated Chi square:

$\mathrm{X} 2=(2163-2081) 2+(1934-1059) 2+(977-1282) 2+(1109-1027) 2=805.3$

$2081 \quad 1059 \quad 1282 \quad 1027$

Chi square analysis: $\mathrm{df}=1$, and $\mathrm{p}=0.005$; the chart value is 7.8

The calculated chi square value was greater than the chart value. There is $99.5 \%$ probability that one can reject the null hypothesis and accept that patients receiving premixed parenteral nutrition will have serum electrolytes, phosphate, and magnesium values that are within normal limits.

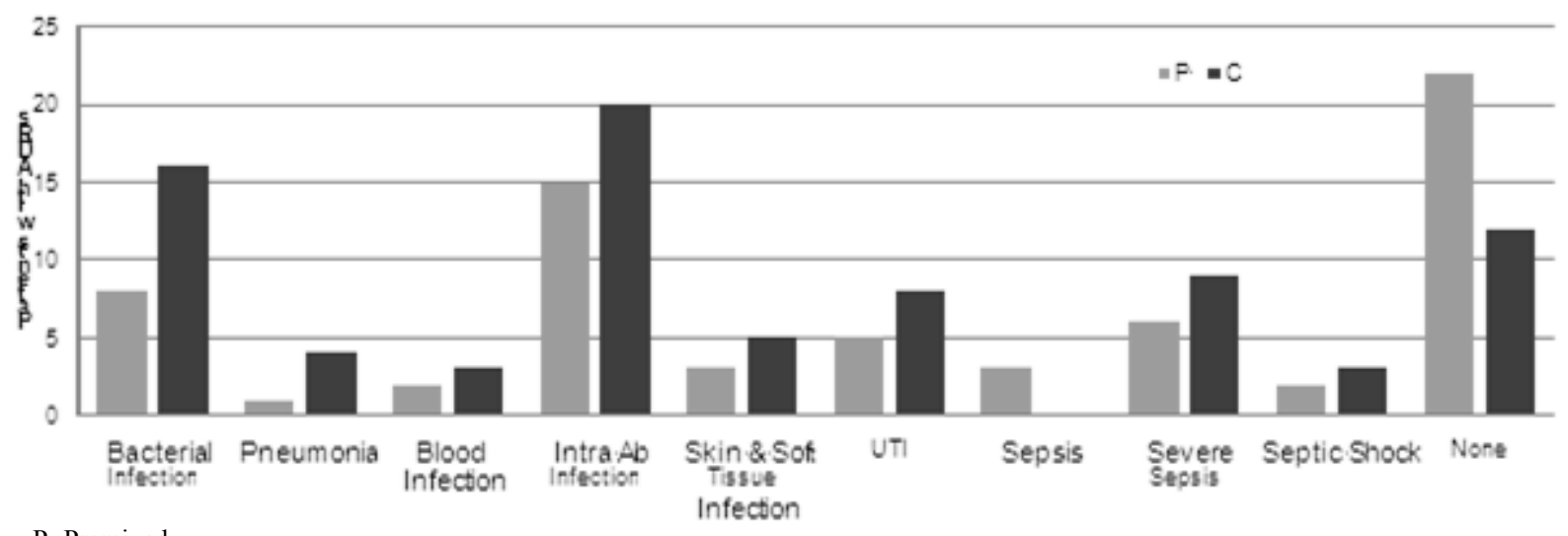

$\mathrm{P}=$ Premixed

$\mathrm{C}=$ Customized

$\mathrm{Ab}=$ abdominal

$\mathrm{UTI}=$ urinary tract infection

Figure 1. Adverse Drug Reactions in Patients Receiving Premixed versus Customized Parenteral Nutrition 


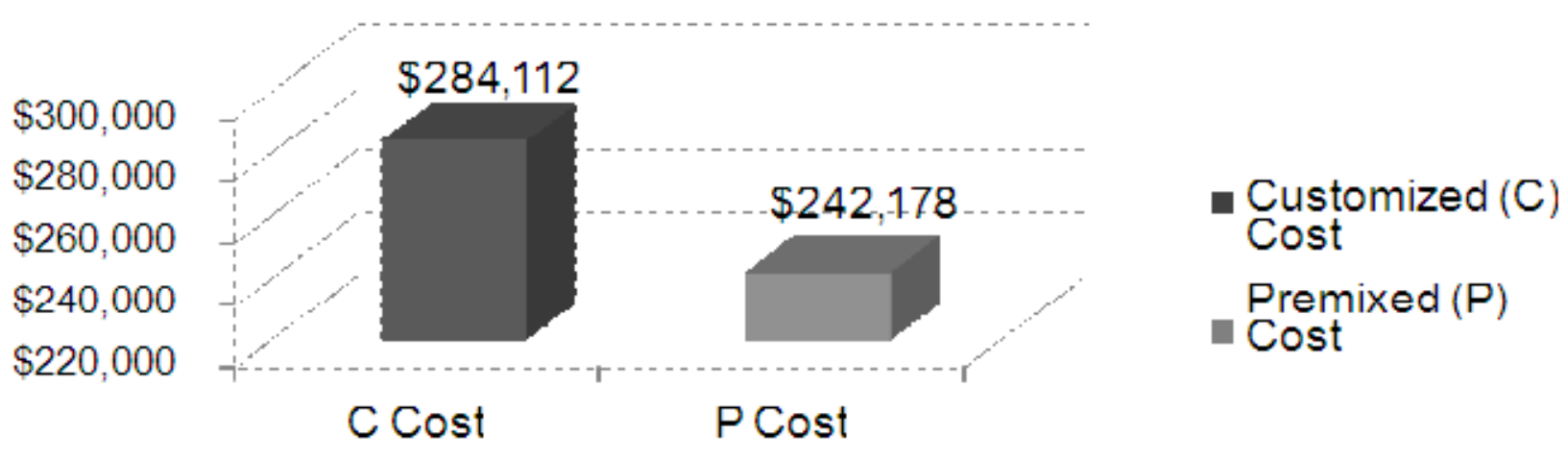

Figure 2. Total Annual System Cost 1-Year Projection*

\section{Limitations}

A few limitations should be considered in interpreting the findings of this research. First, efficacy was measured by serum electrolytes, calcium, phosphate, and magnesium values that were within normal limits and the increasing trend in prealbumin. While these factors are important, they are not the only measure of efficacy in regard to nutrition support. Second, a limitation of this study is the sample size of 50 patients per group. While few large scale studies have been conducted comparing commercially prepared premixed formulations to customized ones, caution should be used when trying to generalize findings of our study alone to broader patient populations. Finally, results of the cost analysis should be interpreted with caution. Due to the limited availability of cost data, only hospital's charges are reported, and savings refer to the charge that the hospital incurred for ingredients of the formulation.

\section{Discussion}

Current literature supports the usefulness of premixed parenteral nutrition in the management of nutritional intake in patients who require parenteral nutrition. For example, a non-randomized study published in $\mathrm{P} \& \mathrm{~T}$ suggests there may be improved electrolyte management in patients receiving standardized PN. [3] It is reasonable to suggest that many patients can be optimally maintained on a premixed PN solution. However, due to the lack of randomized studies, like the Hayes et al study and others that have followed, it has been difficult for many clinicians to have confidence in the results of these studies. Our study contributes to the available literature by providing a strong study design being a prospective randomized controlled trial. Benefits of using premixed PN are decreased risk of microbial contamination, decreased risk of medication errors due to compounding, lower costs, and decreased utilization of Metabolic Support Service Personnel and as a result, a more appropriate utilization of valuable healthcare resources.

\section{Conclusion}

This was a randomized controlled trial of 100 patients requiring central PN. Fifty subjects were prospectively observed under the current customized PN prescription format and 50 subjects were observed with a premixed PN solution. In our study, premixed parenteral nutrition was shown to be equally safe, efficacious and less costly than customized PN. Additionally, patients receiving premixed $\mathrm{PN}$ experienced fewer adverse drug reactions and metabolic abnormalities compared to patients receiving customized parenteral nutrition. Potential cost savings were also actualized with the premixed parenteral nutrition formulas resulting in an average annual savings of $\$ 41,934$.

PN solutions are used by physicians to ensure adequate delivery of calories and protein when patients are unable to receive sufficient nutrition by either the oral route or via feeding tube. In the last two decades, there has been a significant degree of controversy regarding the use of standardized commercially prepared parenteral solutions in the United States. Opponents of customized PN claim that individualized parenteral nutrition is necessary because the critically ill patient requires daily manipulations of macro and micronutrients secondary to metabolic stress and numerous disease states. This study has shown that premixed $\mathrm{PN}$ is a viable option for critically ill patients. Premixed PN solutions were equally safe and effective while being less costly when compared to customized PN formulas.

\section{Acknowledgements}

The authors of this article would like to acknowledge the organization Baxter for their support in the creation of this manuscript.

\section{REFERENCES}

[1] The Joint Commission. www.jcaho.org. Accessed June 30, 2012.

[2] Kochevar M, Guenter P, Holcombe B, Malone A, Mirtallo J. A.S.P.E.N. statement on parenteral nutrition standardization. 
JPEN J Parenter Enteral Nutr. 2007; 31:441-448.

[3] Hayes EM, Oonard BE, Cohen KR, Lauletta J, Ruggiero R. Customized parenteral nutrition solutions: a comparison of serum electrolytes. Pharm Ther. 2000; 25:78-87.

[4] Oxford Parenteral Nutrition Team. Total parenteral nutrition: value of standard feeding regimen. $\mathrm{Br}$ Med J. 1983; 286:1323-1327.

[5] Ruano M, Recuenco I, Torrecilla A, et al. Standard formulas of parenteral nutrition: a study of their use in general hospital. Nutr Hosp. 1993; 8:242-248.

[6] Turpin R, Canada R, Xiaoqing F, Mercaldi C, Pontes-Arruda A, Wischmeyer P. Nutritional therapy cost analysis in the US: premixed multi-chamber bag versus compounded parenteral nutrition. Appl Health Economics Health Pol. 2011; 9:281-292.

[7] Turpin R, Canada T, Rosenthal V, et al. Bloodstream infections association with parenteral nutrition preparations methods in the United States: a retrospective, large database analysis. JPEN J Parenter Enteral Nutr. 2012; 36: 169-176.

[8] DeLegge MH. Parenteral nutrition therapy over the next 5-10 years: where are we heading? JPEN J Parenter Enteral Nutr. 2012; 36:56S-61S.

[9] Maswoswe JJ, Newcomer DR, Quandt CM. Achieving parenteral nutrition cost savings through prescribing guidelines and formulary restrictions. Am J Hosp Pharm. 1987; 44:1376-1381.

[10] Gervasio J. Compounding vs standardized commercial parenteral nutrition product: pros and cons. JPEN J Parenter Enteral Nutr. 2012; 36:40S-41S.

Simmer K, Rakshasbhuvankar A, Deshpande G. Standardized Parenteral Nutrition. Nutrients. 2013 April. 5(4): 1058-1070. doi: 10.3390/nu5041058. 